\title{
Avaliação nutricional de crianças internadas em hospital público e hospital particular, e de suas mães, em Salvador, Bahia
}

\author{
Nutritional assessment of hospitalized children from a public hospital and from private \\ hospital, and of their mothers, in Salvador, Bahia
}

\begin{abstract}
Isabela Tavares Ribeiro ${ }^{1}$, Luciana Rodrigues Silva², Amanda Cunha Kachimareck ${ }^{1}$, Fábio Franco de Freitas Nunes ${ }^{1}$, Carlos Maurício Cardeal Mendes ${ }^{3}$, Ângela Peixoto de Mattos ${ }^{4}$, Gilton Marques dos Santos ${ }^{1}$

${ }^{1}$ Acadêmico de Medicina. Faculdade de Medicina da Bahia, UFBA; ${ }^{2}$ Professora Titular de Pediatria. Faculdade de Medicina da Bahia, UFBA; ${ }^{3}$ Professor de Bioestatística e Epidemiologia. Programa de Pós-Graduação Processos Interativos dos Órgãos e Sistemas, UFBA; ${ }^{4}$ Professora Adjunto de Pediatria. Faculdade de Medicina da Bahia, UFBA
\end{abstract}

\begin{abstract}
Resumo
Introdução: A avaliação nutricional é um eficiente indicador das condições gerais de saúde da criança, especialmente durante a hospitalização. Entretanto, ela é pouco relatada na literatura. Objetivo: Avaliar o estado nutricional de crianças internadas em um hospital público e um hospital particular na Bahia. Metodologia: Trata-se de um estudo tipo coorte transversal, pelo qual foram avaliados os Índices de Massa Corporal (IMC) de 100 crianças internadas em um hospital público e 77 em um hospital particular, bem como de suas respectivas mães, no dia da admissão, entre Junho e Agosto de 2013. Foi utilizada a curva de crescimento Idade x IMC (z-score). Resultados: Das 177 crianças, 65 apresentaram IMC alterado, quatro delas com magreza acentuada, 12, magreza, 24 apresentaram risco de sobrepeso, 19 tinham sobrepeso e seis eram obesas. $O$ risco de sobrepeso e sobrepeso foram as alterações mais frequentes no hospital público e o risco de sobrepeso, a mais frequente no hospital particular. Total de $56,3 \%$ das crianças acima do peso apresentaram mães igualmente acima do peso, enquanto que a concordância de eutrofia entre filhos e suas mães foi de 58,9\%. Conclusão: Risco de sobrepeso foi a alteração mais frequente, no geral, o que está de acordo com a atual situação de transição nutricional do país. Parece haver associação entre estado nutricional da criança e das suas mães. Há necessidade de avaliação nutricional sistemática em todas as crianças hospitalizadas.
\end{abstract}

Palavras-chave: Avaliação nutricional. Estado nutricional. Criança hospitalizada. Imagem corporal.

\begin{abstract}
Introduction: Nutritional assessment is an efficient indicator of general health condition of the children, especially during hospitalization. However, it is less registered in literature. Objective: To acess nutritional status of hospitalized children in a public hospital and in a private hospital at Bahia. Methodology: This was a transversal cohort study, in which the Body Mass Index (BMI) from 100 hospitalized children at a public hospital and from 77 hospitalized children at private hospital was evaluated. The same thing also happened with their respective mothers at the day of admission, between June and August 2013. We used the Age x BMI growth curve (z-score). Results: Among the 177 children, 65 presented alterations on their BMI, four of them with severe underweight, 12, underweight, 24 presented overweight risk, 19 were overweight and six were obese. Overweight and overweight risk were the most frequent alterations at the public and overweight risk were the most frequent alteration at the private hospital. An amount of 56.3\% of all the overweight children had their mothers equally overweight, while the concordance of eutrophic between kids and mothers were 58.9\%. Conclusions: Overweight risk was the most frequent alteration, in general, and this was consistent with the nutritional transition state of our country. It seems that it could exists an association between children's nutritional status and mothers' nutritional status. It's necessary to make a systematic nutritional assessment in all of the hospitalized children.

Keywords: Nutritional assessment. Nutritional status. Hospitalized child. Body Image.
\end{abstract}

\section{INTRODUÇÃO}

A má nutrição corresponde a um desequilíbrio na ingesta de nutrientes essenciais para o organismo ${ }^{1}$. Trata-se de um termo que inclui a obesidade, o sobrepeso, a desnutrição e várias deficiências nutricionais. Tais alterações podem ser identificadas e agravadas em crianças hospitalizadas.

A má nutrição infantil é um problema de saúde pública, com grande impacto físico, econômico e social ${ }^{2,3}$. A

Correspondente / Corresponding: Gilton Marques. Rua Manoel Barreto, 501 Ed. Plácida, ap. 500 - Graça. CEP. 40.150-360 E-mail: gilton18@ hotmail.com criança desnutrida fica mais suceptível a infecções, devido a deficiências imunitárias, apresenta lentificação no processo de cicatrização de feridas, além de função intestinal reduzida e comprometimento do crescimento e desenvolvimento, o que leva a maior período de internamento e aumento de chance de complicações da doença de base ${ }^{4,5}$. Sabe-se que o número de crianças com desnutrição energético-proteica (DEP), de maneira geral, diminuiu consideravelmente nos últimos 20 anos. $O$ Brasil também acompanhou essa redução ${ }^{6,7}$. Por outro lado, o sobrepeso e obesidade têm apresentado caráter epidêmico e prevalência significativamente crescente tanto nos países desenvolvidos quanto 
naqueles em desenvolvimento, como o Brasil ${ }^{8,9}$. Nota-se, inclusive, uma tendência a aumento do número de crianças hospitalizadas apresentando diagnóstico nutricional de obesidade ou sobrepeso. Crianças obesas hospitalizadas também possuem maior risco de apresentar complicações que implicam maior tempo de hospitalização ${ }^{10}$. Elas têm maior risco de desenvolver anemia, apresentar baixos níveis séricos de vitaminas lipossolúveis, hiperlipidemia, resistência insulínica e hiperglicemia, além de várias complicações sistêmicas a curto e longo prazo ${ }^{11}$.

A avaliação nutricional das crianças pode representar não só o estado de saúde delas, mas também da sua família, uma vez que as crianças compartilham informações genéticas, bem como condições sócio-econômico-ambientais com seus respectivos pais, além de hábitos alimentares e hábitos de vida semelhantes ${ }^{12}$. Admite-se que a interação entre fatores ambientais e genotípicos, à medida que configura-se como determinante das diferentes alterações do estado nutricional, produz efeitos similares nos membros de uma mesma família ${ }^{13}$.

Poucos são os relatos sobre o estado nutricional de crianças hospitalizadas no Brasil. Esse estudo justifica-se pela importância de identificar as possíveis alterações no estado nutricional das crianças hospitalizadas, sendo ação fundamental para o estabelecimento precoce das respectivas intervenções que sejam necessárias a partir desse conhecimento.

\section{METODOLOGIA}

O presente estudo foi desenvolvido no período de três meses, entre Junho e Agosto de 2013. Trata-se de um estudo tipo coorte transversal, no qual foram avaliadas, através da aplicação de questionário, crianças entre 0 e 15 anos, hospitalizadas em dois hospitais de Salvador, um particular e outro público. O Termo de Consentimento Livre e Esclarecido (TCLE) foi assinado pelos responsáveis de todas as crianças incluídas no presente estudo. Foram excluídas as crianças hospitalizadas portadoras de neuropatias, com diagnóstico de osteogênese imperfeita, aquelas internadas em Unidade de Terapia Intensiva (UTI), acamadas sem mobilidade, ou ainda aquelas cujos pais não permitiram a inclusão no estudo. $O$ estudo foi aprovado pelo Comitê de Ética.

Foram coletados dados antropométricos de peso e estatura, a fim de obter o cálculo do IMC, de acordo com as recomendações da OMS, tanto das crianças hospitalizadas quanto de suas respectivas mães. A coleta de tais dados, bem como a aplicação do referido questionário foram realizadas por três estudantes de Medicina da Universidade Federal da Bahia (UFBA), previamente treinados. A balança e o estadiômetro utilizados na aferição do peso e estatura das crianças e seus pais foram aqueles disponíveis em cada hospital (balança analógica da marca Welmy no hospital público, e balança digital da marca Filizolla no hospital particular). Admitiu-se precisão de 100 g para medida de peso e $0,5 \mathrm{~cm}$ para medida de estatura. Foram considerados os seguintes diagnósticos nutricio- nais para crianças de 0 a 15 anos: IMC adequado para a idade $(\leq+1$ e $\geq-2$ escores $z$ ); obesidade ( $>3$ escores $z)$; sobrepeso ( $\leq+3$ e $\geq+2$ escores $z$ ); risco de sobrepeso $(\leq+2$ e $>+1$ escores $z)$; magreza ( $<-2$ e $\geq-3$ escores $z)$, magreza acentuada ( $<-3$ escores $z$ ). Por outro lado, foram considerados os seguintes diagnósticos nutricionais para as mães das crianças (adultos): eutrófico (18,5-25); obesidade (acima de 30 ); sobrepeso ( $25-30$ ); baixo peso (inferior a 18,5), segundo os critérios da Organização Mundial de Saúde.

Dividiram-se quatro grupos, a fim de alocar as crianças segundo critérios de faixa etária. $O$ Grupo $A(G A)$ correspondeu a crianças entre 0 a 1 ano; o Grupo B (GB), entre 1 a 5 anos; o Grupo $C$ (GC) composto por crianças de 6 a 10 anos; e o Grupo D (GD) referente a crianças acima de 10 anos de idade.

Por tratar-se de plano amostral não probabilístico, portanto uma amostra propositiva e de conveniência, não foram calculadas estatísticas inferenciais, devido à impossibilidade de uma estimativa adequada do erro padrão. Foram obtidas as estatísticas descritivas com média, mediana, desvio-padrão, quartís para as variáveis mensuradas em escala quantitativa e proporções para as variáveis qualitativas. Como medida de associação epidemiológica, calculou-se a razão de prevalência. Foram calculadas as proporções de crianças do hospital público e privado, conforme a sua classificação do IMC, de modo que a comparação de ambas foi realizada diretamente através das frequências relativas obtidas. Para a comparação entre o julgamento das mães quanto ao estado nutricional de seus filhos e os seus verdadeiros estados nutricionais, foi empregado o índice kappa. As análises foram efetuadas no pacote estatístico, $R$ versão 2.15.2, na plataforma Ubuntu 12.10 .

\section{RESULTADOS}

Foram aplicados 177 questionários, 100 deles em um hospital público, outros 77, em hospital particular, no período estabelecido. No hospital público, foram obtidos dados de 55 meninas e 45 meninos, enquanto que no hospital particular, foram obtidos dados de 35 meninas e 42 meninos, totalizando, juntos, 90 meninas, e 87 meninos. A média de idade das crianças foi de 4,8 anos (desvio padrão: 4,2).

O GA englobou 27 (15,3\%) crianças, das quais 13 delas estavam internadas em hospital público, e 14, em hospital particular. O GB, por sua vez, foi composto por $96(54,2 \%)$ crianças, 46 delas em hospital público e 50 em hospital particular. Já o GC ficou constituído por 28 $(15,8 \%)$ crianças, dentre elas 19 internadas em hospital público e 9 em hospital particular. Finalmente, o GD foi representado por $26(14,7 \%)$ crianças, das quais 22 estavam internadas em hospital público e 4 em hospital particular. Nota-se que, para os dois hospitais, o GB englobou o maior número de crianças, correspondendo a $46 \%$ de todas as crianças do hospital público e a $64.9 \%$ de todas as crianças do hospital particular (Tabela 1). 
A partir dos dados do IMC das crianças internadas, foram obtidos os seguintes diagnósticos nutricionais, referentes aos dois hospitais: 112 (63,3\%) das crianças apresentaram IMC adequado para a idade, quatro $(2,3 \%)$ delas teve diagnóstico de magreza acentuada, doze $(6,8 \%)$ apresentaram magreza, $24(13,6 \%)$ apresentaram risco de sobrepeso, 19 (10,7\%) delas apresentaram sobrepeso, e finalmente, seis $(3,4 \%)$ foram consideradas obesas. Risco de sobrepeso foi o a alteração mais frequente encontrada. Os diagnósticos nutricionais referentes a cada hospital estão representados na Tabela 1.

Nota-se que, dentre as alterações nos diagnósticos nutricionais das crianças internadas em hospital público, risco de sobrepeso e sobrepeso foram os mais prevalentes, totalizando 20 crianças, que correspondeu cada uma com $10,0 \%$ do total. O risco de sobrepeso foi também a alteração do diagnóstico nutricional mais prevalente dentre as crianças internadas em hospital particular, correspondendo a $14(18,2 \%)$ crianças.

Quando estratificadas por faixa etária (Tabela 1), nota-se que o GB apresentou o maior número de crianças com diagnóstico nutricional alterado, tanto para crianças internadas em hospital público, quanto para aquelas internadas em hospital particular.

Tabela 1 - Diagnósticos nutricionais de crianças internadas em hospital público e particular

\begin{tabular}{|c|c|c|c|c|c|c|c|c|c|c|}
\hline \multirow{2}{*}{$\begin{array}{l}\text { Diagnóstico nutri- } \\
\text { cional }\end{array}$} & \multicolumn{5}{|c|}{ Hospital Público } & \multicolumn{5}{|c|}{ Hospital Particular } \\
\hline & GA & GB & $\mathrm{GC}$ & GD & TOTAL & GA & GB & GC & GD & TOTAL \\
\hline Adequado & $\begin{array}{c}7 \\
(53,8 \%)\end{array}$ & $\begin{array}{c}30 \\
(65,2 \%)\end{array}$ & $\begin{array}{c}14 \\
(73,7 \%)\end{array}$ & $\begin{array}{c}11 \\
(50,0 \%)\end{array}$ & 62 & $\begin{array}{c}11 \\
(78,6 \%)\end{array}$ & $\begin{array}{c}30 \\
(60 \%)\end{array}$ & $\begin{array}{c}6 \\
(66,7 \%)\end{array}$ & $\begin{array}{c}3 \\
(75,0 \%)\end{array}$ & 50 \\
\hline Magreza acentuada & $\begin{array}{c}2 \\
(15,4 \%)\end{array}$ & $\begin{array}{c}1 \\
(2,2 \%)\end{array}$ & $\begin{array}{c}0 \\
(0,0 \%)\end{array}$ & $\begin{array}{c}1 \\
(4,5 \%)\end{array}$ & 4 & $\begin{array}{c}0 \\
(0,0 \%)\end{array}$ & $\begin{array}{c}0 \\
(0,0 \%)\end{array}$ & $\begin{array}{c}0 \\
(0,0 \%)\end{array}$ & $\begin{array}{c}0 \\
(0,0 \%)\end{array}$ & 0 \\
\hline Magreza & $\begin{array}{c}1 \\
(7,7 \%)\end{array}$ & $\begin{array}{c}2 \\
(4,3 \%)\end{array}$ & $\begin{array}{c}1 \\
(5,3 \%)\end{array}$ & $\begin{array}{c}5 \\
(22,7 \%)\end{array}$ & 9 & $\begin{array}{c}1 \\
(7,4 \%)\end{array}$ & $\begin{array}{c}2 \\
(4 \%)\end{array}$ & $\begin{array}{c}0 \\
(0,0 \%)\end{array}$ & $\begin{array}{c}0 \\
(0,0 \%)\end{array}$ & 3 \\
\hline Risco de sobrepeso & $\begin{array}{c}2 \\
(15,4 \%)\end{array}$ & $\begin{array}{c}4 \\
(8,7 \%)\end{array}$ & $\begin{array}{c}1 \\
(5,3 \%)\end{array}$ & $\begin{array}{c}3 \\
(13,6 \%)\end{array}$ & 10 & $\begin{array}{c}2 \\
(14,3 \%)\end{array}$ & $\begin{array}{c}11 \\
(22 \%)\end{array}$ & $\begin{array}{c}1 \\
(11.1 \%)\end{array}$ & $\begin{array}{c}0 \\
(0,0 \%)\end{array}$ & 14 \\
\hline Sobrepeso & $\begin{array}{c}1 \\
(7,7 \%)\end{array}$ & $\begin{array}{c}5 \\
(10,7 \%)\end{array}$ & $\begin{array}{c}3 \\
(15,8 \%)\end{array}$ & $\begin{array}{c}1 \\
(4,5 \%)\end{array}$ & 10 & $\begin{array}{c}0 \\
(0,0 \%)\end{array}$ & $\begin{array}{c}6 \\
(12 \%)\end{array}$ & $\begin{array}{c}2 \\
(22,2 \%)\end{array}$ & $\begin{array}{c}1 \\
(25,0 \%)\end{array}$ & 9 \\
\hline Obesidade & $\begin{array}{c}0 \\
(0,0 \%)\end{array}$ & $\begin{array}{c}4 \\
(8,7 \%)\end{array}$ & $\begin{array}{c}0 \\
(0,0 \%)\end{array}$ & $\begin{array}{c}1 \\
(4,5 \%)\end{array}$ & 5 & $\begin{array}{c}0 \\
(0,0 \%)\end{array}$ & $\begin{array}{c}0 \\
(0,0 \%)\end{array}$ & $\begin{array}{c}0 \\
(0,0 \%)\end{array}$ & $\begin{array}{c}0 \\
(0,0 \%)\end{array}$ & 0 \\
\hline TOTAL & $\begin{array}{c}13 \\
(100 \%)\end{array}$ & $\begin{array}{c}46 \\
(100 \%)\end{array}$ & $\begin{array}{c}19 \\
(100 \%)\end{array}$ & $\begin{array}{c}23 \\
(100 \%)\end{array}$ & 100 & $\begin{array}{c}14 \\
(100 \%)\end{array}$ & $\begin{array}{c}49 \\
(100 \%)\end{array}$ & $\begin{array}{c}9 \\
(100 \%)\end{array}$ & $\begin{array}{c}4 \\
(100 \%)\end{array}$ & 77 \\
\hline
\end{tabular}

Legenda: GA (Grupo A: 0 a 1 ano); GB (Grupo B: 1 a 5 anos); GC (Grupo C: 6 a 10 anos); GD (Grupo D: acima de 10 anos).

A partir de uma análise mais simplificada, destaca-se que, para os dois hospitais, dentre as crianças com diagnóstico alterado, 49 (75,4\%) delas estavam acima do peso, o hospital público com 25 dessas crianças, enquanto que o hospital particular com 24 delas. Por outro lado, um total de $16(24,6 \%)$ crianças com diagnóstico nutricional alterado estava abaixo do peso ideal, sendo que 13 delas estavam internadas no hospital público e apenas três delas no hospital particular.

Quando comparados os diagnósticos nutricionais das crianças internadas com suas respectivas mães (Tabela 2 ), nota-se que $56,3 \%$ das crianças diagnosticadas acima do peso ideal para idade possuíam mães igualmente acima do peso, enquanto que $58,9 \%$ das crianças classificadas com peso ideal para a idade também possuíam respectivas mães com peso adequado. Não foi possível realizar associação entre crianças abaixo do peso ideal para a idade e respectivas mães com baixo peso, pois nenhuma das mães entrevistadas apresentou IMC abaixo do normal.

Tabela 2 - Comparação entre diagnósticos nutricionais das crianças e de suas respectivas mães

\begin{tabular}{l|c|c|c|}
\hline & $\begin{array}{l}\text { Acima do peso } \\
\text { (mães) }\end{array}$ & $\begin{array}{l}\text { Peso normal } \\
\text { (mães) }\end{array}$ & TOTAL \\
\hline $\begin{array}{l}\text { Peso normal } \\
\text { (filhos) }\end{array}$ & $\begin{array}{c}46 \\
(41,1 \%)\end{array}$ & $\begin{array}{c}66 \\
(58,9 \%)\end{array}$ & $\begin{array}{c}112 \\
(100 \%)\end{array}$ \\
\hline Abaixo do peso & 10 & 6 & 16 \\
(filhos) & $(62,5 \%)$ & $(37,5 \%)$ & $(100 \%)$ \\
\hline Acima do peso & 27 & 21 & 48 \\
(filhos) & $(56,3 \%)$ & $(43,7 \%)$ & $(100 \%)$ \\
\hline
\end{tabular}


Verificou-se que, nos dois hospitais, a maioria das mães 112 (63,3\%), quando perguntadas a respeito da sua percepção sobre a adequação do peso de seus filhos, declararam que eles estavam com peso adequado. No hospital público, 62 (62\%) mães julgaram seus filhos com pesos adequados, enquanto que, no hospital particular, 50 (64,9\%) mães igualmente admitiram que seus filhos estavam com peso adequado. Na análise de concordância entre a opinião das mães sobre a adequação do peso de seus filhos e os respectivos diagnósticos nutricionais, observou-se que, para os dois hospitais, houve concordância de acerto para resposta positiva em 69 (66,3\%) dos 104 casos diagnosti- cados como peso adequado. Por outro lado, 43 (58,9\%) das 73 crianças classificadas com diagnóstico nutricional alterado foram julgadas pelas respectivas mães com peso adequado. Os resultados obtidos referentes a essa análise para cada hospital estão representados na Tabela 3.

Nota-se que, no hospital público, $57,4,0 \%$ das crianças classificadas com diagnóstico nutricional alterado foram julgadas pelas mães como com peso adequado. No hospital particular, essa mesma relação foi ainda maior, de 61,5\%. Os valores de kappa (Tabela 3) demonstraram ausência de concordância global, tanto no hospital público como no hospital particular.

Tabela 3 - Concordância entre de diagnóstico nutricional das crianças e percepção das respectivas mães no hospital público e particular

\begin{tabular}{|c|c|c|c|c|c|c|}
\hline \multirow[b]{2}{*}{ Concordância } & \multicolumn{3}{|l|}{ Hospital público } & \multicolumn{3}{|l|}{ Hospital particular } \\
\hline & $\begin{array}{l}\text { Percepção da } \\
\text { mãe: } \\
\text { Adequado }\end{array}$ & $\begin{array}{c}\text { Percepção da } \\
\text { mãe: } \\
\text { Alterado }\end{array}$ & TOTAL & $\begin{array}{l}\text { Percepção da } \\
\text { mãe: } \\
\text { Adequado }\end{array}$ & $\begin{array}{l}\text { Percepção da } \\
\text { mãe: } \\
\text { Alterado }\end{array}$ & TOTAL \\
\hline Adequado & $\begin{array}{c}35 \\
(66 \%)\end{array}$ & $\begin{array}{c}18 \\
(34 \%)\end{array}$ & $\begin{array}{c}53 \\
(100 \%)\end{array}$ & $\begin{array}{c}34 \\
(66,6 \%)\end{array}$ & $\begin{array}{c}17 \\
(33,3 \%)\end{array}$ & $\begin{array}{c}51 \\
(100 \%)\end{array}$ \\
\hline Alterado & $\begin{array}{c}27 \\
(57,4 \%)\end{array}$ & $\begin{array}{c}20 \\
(42,6 \%)\end{array}$ & $\begin{array}{c}47 \\
(100 \%)\end{array}$ & $\begin{array}{c}16 \\
(61,5 \%)\end{array}$ & $\begin{array}{c}10 \\
(38,5 \%)\end{array}$ & $\begin{array}{c}26 \\
(100 \%)\end{array}$ \\
\hline
\end{tabular}

NOTA: kappa hospital público: 0,087 (8,7\%); kappa hospital particular: 0,088 $(8,8 \%)$

\section{DISCUSSÃO}

Este estudo definiu as taxas de alterações na avaliação nutricional de crianças internadas em um hospital público e um hospital particular em Salvador, Bahia e determinou que muitas dessas crianças apresentaram diagnósticos nutricionais alterados, estando, consequentemente, submetidas a maior risco de complicações intra-hospitalares, bem como maior período de internamento, o que demonstra possibilidade de agravos em curto e longo prazo.

Os pacientes deste estudo, em conjunto, formaram um grupo desigual, com idades bastante distintas, tendo sido, por este motivo, estratificadas em grupos de faixas etárias. O maior número de pacientes pertenceu ao grupo de faixa etária entre 1 e 5 anos (54,2\%), seguido do grupo de crianças entre 6 e 10 anos $(15,8 \%)$, menores de 1 ano (15,3\%) e maiores que 10 anos (14,7\%). Em geral, percebe-se que as internações foram mais frequentes entre crianças e lactentes do que entre adolescentes. Outro estudo realizado em São Paulo ${ }^{14}$ também revela relação similar, com $69,0 \%$ de internações de crianças e lactentes e 31,0\% de internações de adolescentes. O GB (1- 5 anos) foi o grupo responsável pelo maior número de alterações nos diagnósticos nutricionais das crianças avaliadas. Porém, não podemos afirmar que isso seja devido a uma maior vulnerabilidade das crianças pertencentes a essa faixa etária, pois pode representar apenas reflexo da maior quantidade de crianças encontradas nesse grupo.

Poucos estudos foram realizados a fim de demonstrar o perfil nutricional de crianças hospitalizadas. Um estudo realizado no Brasil, em $2008^{15}$, demonstrou que $21,8 \%$ das crianças na admissão estavam abaixo do peso, enquanto que $13,8 \%$ estavam acima do peso ou obesas. Entretanto, o presente estudo revelou uma relação inversa, onde apenas 9,0\% do total das crianças hospitalizadas na admissão de ambos os hospitais estavam abaixo do peso ideal, enquanto que $27,7 \%$ das crianças estavam acima do peso. Essa divergência de resultados pode refletir a ausência de uma definição categórica para as alterações do estado nutricional, em virtude dos diversos índices disponíveis para sua avaliação, com diferentes pontos de corte. Outra possibilidade é devida à influência dos pacientes internados em hospital particular no presente estudo, uma vez que Silveira et al. avaliaram apenas crianças internadas em um hospital público, assim como a maioria dos estudos similares o fazem. De fato, dados da $\mathrm{OMS}^{8}$ revelam que as taxas de sobrepeso e obesidade infantil têm crescido de forma significante nas últimas décadas, ao contrário das taxas de desnutrição, que vêm decrescendo. O perfil dos pacientes pediátricos internados no presente estudo foi concordante com a atual transição nutricional do país

Alguns estudos revelam que o estado nutricional das mães tem influência sobre o estado nutricional de seus filhos ${ }^{12,13,16}$. Neste estudo, foi demonstrado que a maioria das crianças $(56,3 \%)$ acima do peso ideal para idade possuíam mães igualmente acima do peso. Da mesma forma, a maioria das crianças (58,9\%) eutróficas também possuíam respectivas mães com peso adequado. Ainda, genericamente, $57,8 \%$ das crianças que apresentaram alguma alteração no diagnóstico nutricional possuíam 
mães acima do peso ideal. Um estudo realizado no Rio de Janeiro, em $1996^{13}$, revelou que crianças com sobrepeso tinham maiores chances de ter mães com sobrepeso, sugerindo relação direta entre estado nutricional materno e alteração no diagnóstico nutricional das crianças. Os resultados do presente estudo foram semelhantes, ratificando essa conclusão. Outro estudo similar publicado em $1999^{12}$ revelou relação direta entre déficit estatural (importante indicador de desnutrição infantil) e baixo peso materno. Esta última relação não foi obtida neste estudo, uma vez que não foram encontradas mães com baixo peso.

A análise de concordância entre o julgamento das mães a respeito do estado nutricional dos respectivos fiIhos e seus verdadeiros diagnósticos nutricionais revelou importante alteração na percepção de inadequação nutricional. Tanto as mães cujos filhos estavam internados em hospital público quanto aquelas provenientes de hospital particular, em sua maioria, julgaram como "adequado" o peso de seus filhos diagnosticados com alguma alteração nutricional. Inclusive, a frequência desse tipo de erro foi maior nas mães provenientes de hospital particular. Um estudo semelhante realizado no Espírito Santo, Brasil, em $2009^{17}$, mostrou que $63,0 \%$ das crianças acima do peso foram consideradas pelas respectivas mães com peso adequado. Outro estudo publicado em 2002, em Campinas, revelou que $63,3 \%$ das mães de crianças com baixo peso não reconheceram essa condição, bem como $86,4 \%$ das mães de crianças com sobrepeso também não o fizeram ${ }^{18}$. Nossos resultados não permitiram a comparação entre o tipo de alteração percebido pelas mães e o real estado nutricional de seus filhos, pois a pergunta realizada no questionário não foi específica para todos os diagnósticos nutricionais possíveis.

A percepção errônea das mães sobre o estado nutricional dos seus filhos pode ter consequências negativas no crescimento e desenvolvimento dessas crianças. $O$ estilo de vida e hábitos alimentares (qualidade e quantidade de alimento) das crianças são basicamente dependentes das escolhas e hábitos de suas mães e seus pais. Elas são feitas justamente a partir das percepções sobre o estado nutricional de seus filhos ${ }^{19}$. Logo, esse erro pode ser fator determinante para a perpetuação de uma condição nutricional inadequada dessas crianças.

Novos estudos necessitam ser realizados a fim de melhor caracterizar as crianças hospitalizadas cujos diagnósticos nutricionais encontram-se alterados, a fim de dar subsídios para a questão em pauta, além de divulgar a necessidade e importância da sistematização da avaliação nutricional entre os profissionais de saúde para todas as crianças hospitalizadas.

\section{Agradecimentos:}

Agradecemos aos pequenos pacientes que nos ajudaram a realizar este trabalho, e aos seus respectivos pais, por permitirem a inclusão de seus filhos na pesquisa.

\section{REFERÊNCIAS}

1. KRUIZENGA, H.M. et al. Effectiveness and cost-effectiveness of early screening and treatment of malnourished patients. Am. J. Clin. Nutr., Bethesda, v. 82, n. 5, p. 1082-1089, nov 2005.

2. WAITZBERG, D.L.; CAIAFFA, W.T.; CORREIA, M.I. Hospital malnutrition: the Brazilian national survey (IBRANUTRI): a study of 4000 patients. Nutrition., Paris, v. 17, n. 7-8, p. 573-580, 2001.

3. CORREIA, M.IT.D.; WAITZBERG, D.L. The impact of malnutrition on morbidity, mortality, length of hospital stay and costs evaluated through a multivariate model analysis. Clin. Nutr., Edinburgh, v. 22, n. 3, p. 235-239, 2003.

4. JOOSTEN, K.F. et al. National malnutrition screening days in hospitalised children in The Netherlands. Arch. Dis. Child., London, v. 95, n. 2, p. 141-145, Feb. 2010.

5. PAWELLEK, I.; DOKOUPIL, K.; KOLETZKO, B. Prevalence of malnutrition in paediatric hospital patients. Clin. Nutr., Edinburgh, v. 27, n. 1, p. 72-76, Feb. 2008.

6. DE ONIS, M.; FRONGILLO, E.A.; BLÖSSNER, M. Is malnutrition declining? An analysis of changes in levels of child malnutrition since 1980. Bull. World Health Organ., Geneve, v. 78, n. 10, p. 1222-1233, Jan. 2000.

7. BEMFAM. Sociedade Civil Bem-Estar Familia no Brasil. Pesquisa Nacional sobre Demografia e Saúde. Rio de Janeiro. 1997.

8. WHO. World Health Organization. Growth reference data for 5-19 years. 2007. Disponível em: <http://www.who.int/growthref/en/>. Acesso em: Jun 2013.

9. BRASIL. Ministério da Saúde. Portal da Saúde - www.saude.gov. br [Internet]. Disponível em:<http://portalsaude.saude.gov.br/portalsaude/>. Acesso em: 27 jan. 2013.

10. WANG, G.; DIETZ, W.H. Economic burden of obesity in youths aged 6 to 17 years: 1979-1999. Pediatrics., Evanston, v. 109, n. 5, p. E81-E81, May 2002

11. CALI, A.M.G.; CAPRIO, S. Obesity in children and adolescents. J. Clin. Endocrinol. Metab., Philadelphia, v. 93, Suppl 1, p. S31-S36, Nov 2008.

12. ENGSTROM, E.M.; ANJOS, L.A. Déficit estatural nas crianças brasileiras: relação com condições sócio-ambientais e estado nutricional materno. Cad. Saude Publica., Rio de Janeiro, v. 15, n. 3, p. 559-567, 1999.

13. ENGSTRON, E.M.; ANJOS, L.A. Relationship between maternal nutritional status and overweight in Brazilian children. Rev. Saúde Pública., São Paulo, v. 30, n. 3, p. 233-239, 1996.

14. SIMÕES, A.P.B. et al. Estado nutricional de crianças e adolescentes hospitalizados em enfermaria de cirurgia pediátrica. Rev. Paul. Pediatr., São Paulo, v. 28, n. 1, p. 41-47, Mar 2010.

15. DE MORAES SILVEIRA, C.R. et al. Evolution of nutritional status of pediatric in patients of a tertiary care general hospital in Brazil. Nutr. Hosp., Madrid, v. 23, n. 6, p. 599-606, 2008.

16. NOBREGA, F.J. et al. Nutritional conditions of mothers and sons: relation with the weight at born, maternals and socioeconomics variable. J. Pediatr. Rio de Janeiro, v. 67, n. 9-10, p. 288-296, 1991.

17. MOLINA, M.C.B. et al. Correspondence between children's nutritional status and mothers' perceptions: a population-based study. Cad. Saúde Pública, Rio de Janeiro, v. 25, n. 10, p. 2285-2290, Oct. 2009.

18. CARVALHAES, M.A.B.L.; GODOY, I. As mães sabem avaliar adequadamente o peso das crianças? Rev. Nutr., Campinas, v. 15, n. 2, p. 155-162, 2002.

19. ETELSON, D. et al. Childhood obesity: do parents recognize this health risk? Obes. Res., Baton Rouge, v. 11, n. 11, p. 1362-1368, Dec. 2003.

Submetido em: 06.11.2014

Aceito em: 11.12.2014 